\title{
Historia
}

\section{Primera médica peruana, Dra. Laura Esther Rodríguez Dulanto (1872 - 1919)}

\author{
Héctor Díaz ${ }^{1,2}$
}

\begin{abstract}
Resumen
Es meritorio recordar y rendir merecido homenaje a la Dra. Laura Esther Rodríguez Dulanto, mujer de extraordinaria inteligencia y vocación, quien desoyendo los aventurados prejuicios de la época- pues no había estudios secundarios para señoritas ni estudios universitarios para la mujer peruana-fue la primera mujer en el Perú que ingresó a la Universidad, en mayo de 1892, y finalmente juramentó como Médica Cirujana el 25 de octubre de 1900, en la Facultad de Medicina de la UNMSM, lo cual significó un hito sin precedentes en el Perú y de la medicina peruana, pues fue La Primera Médica Peruana. Además; doy datos inéditos sobre sus verdaderos nombres, la fecha exacta de su nacimiento y del lugar exacto donde nació.
\end{abstract}

Palabrasclave $\quad$ Historia de la medicina, Perú; mujeres; médicos mujeres.

The first peruvian female physician. Laura Esther Rodríguez-Dulanto MD (1872 - 1919) Abstract

It is meritorious to remember and render deserved homage to Laura Esther Rodriguez-Dulanto MD, woman of extraordinary intelligence and vocation, who ignoring the epoch's adventurous prejudices - because young Peruvian ladies could not seek high school or university studies-she was de first woman in Peru to enter the University in may 1892 and finally vowed as Physician and Surgeon on October 25, 1900, at the Faculty of Medicine of San Marcos University. This event did not have precedence in Peru and in Peruvian medicine, as she was The First Peruvian Female Physician. Moreover; I include unpublished data concerning her true names, exact date of birth and birth site.

Key words: History of medicine, Peru; women; physicians, women.

La fecha de nacimiento de la Dra. Laura Esther Rodríguez Dulanto (Figura 1), no concuerda en los relatos anteriores, en donde algunos refieren nació en $1874\left({ }^{1}\right)$; en $1875\left({ }^{2-4}\right)$ y en $1876\left({ }^{5}\right)$. Algunos dieron fechas más precisas de su nacimiento, el 13

Profesor Asociado de la Facultad de Medicina, UNMSM. Lima, Perú. Jefe del Servicio Gastroenterología del Hospital Nacional G. Almenara I. EsSALUD. Lima, Perú. de octubre de $1875\left({ }^{2}\right)$. Respecto al lugar de nacimiento, nació en Supe el antiguo; Distrito Supe de la ex-Provincia Chancay, Departamento Lima; actualmente, está considerado en la Provincia Barranca, del Departamento Lima. Su lugar natal fue destruido por lluvias torrenciales y huaycos, durante el mes de marzo de 1891, y los libros bau-

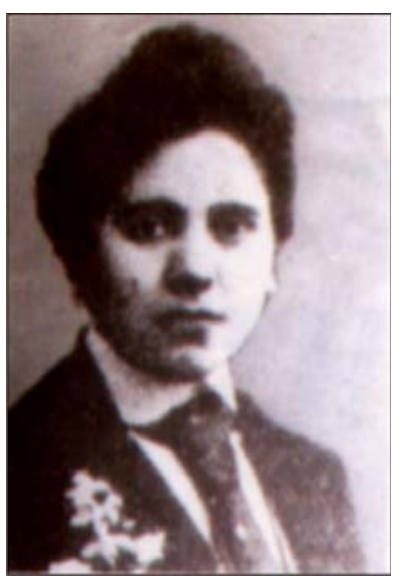

Figura 1. Dra. Laura Esther Rodríguez Dulanto (1872 - 1919). Fuente: Colegio Médico del Perú. Mujeres Médicas ante los Nuevos Retos. Revista Médica. 
tismales de su época fueron derivados a los pueblos de Pativilca, Barranca y Puerto Supe, lo cual dificultó hallar su partida bautismal.

En dicha partida bautismal dice respecto a su nombre: "María Laura Ester, bautizada el 28 de octubre de 1872 , de diez días de nacida, en la Capilla de Santa María Magdalena de Supe, hija legítima de Don Marcelo Rodríguez y de Doña María Cristina...". Los aportes que doy son: 1) Su nombre completo es María Laura Ester Rodríguez Dulanto, 2) Su verdadera fecha de nacimiento: 18 de octubre de 1872; y, 3) Lugar de nacimiento: Supe. Figura 2.

En el Siglo XIX, Supe antiguo estaba ubicado en lo que actualmente se denomina Campiña de Supe, y la doctora nació en la zona denominada actualmente Campiña Baja, pri-

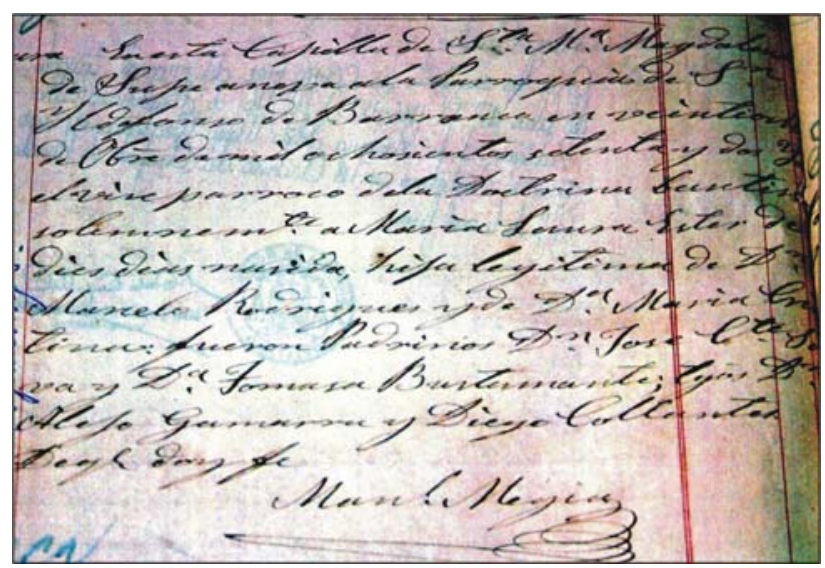

Figura 2. Copia certificada de la partida bautismal de la Dra. María Laura Esther Rodríguez Dulanto, nacida el 18 de octubre de 1872, en Supe. Copia Certificada Abril 28 de 2007.

"En esta Capilla de Sta. Ma. Magdalena de Supe anexa a la Parroquia de Sn. Ildefonso de Barranca en veintiocho de Obre. de mil ochocientos setenta y dos Yo el vise párroco de la Doctrina bautisé solemnemente a María Laura Ester de dies días nasida, hija legítima de Dn. Marcelo Rodríguez y de Da. María Cristina: fueron sus padrinos Dn. José Cte. Serva y Da. Tomasa Bustamente; hijos Dn. Alejo Gamarra y Diego Collantes. Doy la doyfe.

Manuel Mujica." mer lugar del Perú donde por iniciativa de los lugareños y vecinos se proclamó la Independencia Nacional; un lunes 5 de abril de 1819; así lo confirma el Virrey Joaquín de la Pezuela, en sus Memorias de Gobierno.

En Supe antiguo pasó parte de su infancia (Figura 3). Luego, sus padres se vinieron a Lima, en donde estudió primaria, en el colegio Badani $\left({ }^{3}\right)$. Su inteligencia despierta y avivado corazón le permitieron ser precoz preceptora de tercer grado. Fue cuando tomó la firme decisión de no poder dejar de saber más. Su hermano y otros muchachos seguían aprendiendo en la secundaria. No había colegios de secundaria para niñas. En esa época, era sobreentendido y era la regla, que las mujeres con solo los rudimentos de lectura y escritura debían prepararse para el matrimonio. Pero, Laura E. Rodríguez Dulanto era una mujer extraordinaria y se había propuesto ser doctora en Ciencias y en Medicina y nada le amedrentaría. Tercamente, se impuso una meta definitiva: para cuando su hermano Abraham hubiera terminado la secundaria, ella también lo habría hecho. Al respecto, decía: "Mi hermano, al regresar del Colegio Guadalupe, donde estudiaba la secundaria, se prestaba los cuadernos de sus compañeros y me los traía también y durante dos horas me repetiría las lecciones".

Muy joven terminó su instrucción secundaria, pero como en aquel entonces no existían colegios oficiales de instrucción secundaria para señoritas, la Dirección de Instrucción nombró un jurado especial para tomarle el examen $\left({ }^{1,3}\right)$; aprobándolo con nota sobresaliente.

Luego, se preparó con igual persistencia e ingresó, en mayo de 1892, a la Facultad de Ciencias de la Universidad Nacional Mayor de San Marcos, con el calificativo de 20, mereciendo comentarios del diario El Comercio $\left({ }^{1}\right)$, ya que era la primera mujer en el Perú que ingresaba a la Universidad, a la edad de 19 años. En ella no cesaba su espíritu sediento de conocimientos. Aún antes de concluir los estudios en la 
Facultad de Ciencias, se matriculó en la Facultad de Medicina, en el año de 1894; así consta en el Quinto Libro de Matrículas, Página 109. Primer matrícula $\mathrm{N}^{\circ} 3022$. Expediente $\mathrm{N}^{\circ} 38\left(^{5}\right)$. Así fue como ingresó la primera mujer peruana a los estudios médicos, a la edad de 21 años.

Para sus trabajos de anatomía, por entonces con severas restricciones para las mujeres, debió estar detrás de un biombo. A veces preguntaba, y se la comparaba como la de un ciego que quiere reconocer una realidad que no ve. En su casa repasaba con su hermano Abraham, quien también estudiaba medicina, todos los pormenores, como si estuviera en la sala de disección. Por sus altas calificaciones -había sido comentado su caso en los pasillos del Congreso de la República-, una ponencia fue aceptada y por Resolución Legislativa del 15 de diciembre de 1895 el Congreso Nacional le concedió un subsidio de 40 soles mensuales hasta el término de sus estudios. A la vez que cursaba el tercer año, tuvo una autorización especial del Decano de la Facultad de Medicina para realizar disecciones anatómicas en sala separada, en compañía de su hermano Abraham. Tales circunstancias representaron un enorme adelanto y superación para su tiempo.

Su rendimiento fue brillante y a través de sus estudios mereció los primeros premios y las contentas para los grados de Bachiller y Doctorado, según anota Alberto Tauro del Pino en su Enciclopedia Ilustrada del Perú, Lima, $1987\left({ }^{3}\right)$.

Cuando estudiaba el quinto año de medicina, fue la primera mujer en el Perú en obtener el grado de Bachiller en Ciencias, el 27 de octubre de 1898, con calificativo sobresaliente. Su tesis fue sobre "Estudios Geológicos en la Provincia de Chancay" y por su calidad fue publicada en los Anales Universitarios, Tomo XXVI. Este acontecimiento fue publicado al siguiente día en el Diario El Comercio $\left({ }^{6}\right)$.

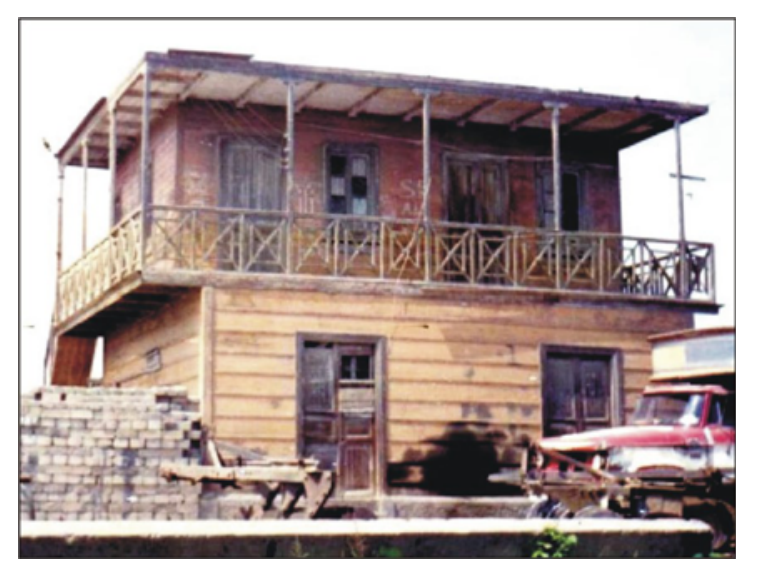

Figura 3. Casa donde nació y vivió su infancia Laura Esther Rodríguez Dulanto, en Supe antiguo, ubicada en la zona actualmente denominada Campiña Baja.

Continuó estudios y prácticas clínicas y de laboratorio en la Facultad de Medicina. Alcanzó el grado de Bachiller en Medicina con la Tesis "Empleo del Ictiol en las Inflamaciones Pelvianas", que lo obtuvo el 23 de diciembre de $1899\left({ }^{3,4,7}\right)$. Y, luego de rendir los exámenes integrales, recibió el Título de Médica Cirujana, por primera vez otorgada a una mujer, con fecha 25 de octubre de $1900\left(^{4}\right)$. Era así la primera mujer que juró la demanda hipocrática en el Perú.

En Lima, en el año 1900, recibe alborozada el advenimiento del nuevo siglo. El alumbrado eléctrico despierta a la ciudad. Pero, otra antorcha se prendió para flamear incesantemente: Laura Esther optó el Título de Médica Cirujana. La mujer peruana puso el fundamento de una gran empresa; la médica peruana. Se hizo presente en la realidad nacional, comenzó el ejercicio de la profesión.

Como Interna de Constantino T. Carva1lo, publicó en la Crónica Médica el trabajo: "Enorme quiste ovárico, acompañado de otro pequeño" (año 1898). Posteriormente, escribió sobre "Fibroma uterino" (año1900) $\left({ }^{3}\right)$.

La trayectoria profesional de Laura Esther Rodríguez Dulanto no fue muy amplia 
longitudinalmente $\left(^{5}\right)$, pero puso las bases de un ejercicio profesional integral. Fue clínica asistencial al lado de los maestros Bello y Corpancho. Tenía el corazón avivado y era solidaria. Así, fundó la Primera Escuela de Enfermería del país $\left({ }^{1,3}\right)$, donde ejerció la docencia, enseñando anatomía, fisiología e higiene, con prácticas en los hospitales Santa Ana y Dos de Mayo. Con el producto de las erogaciones compró un equipo completo de cirugía, que entregó al Hospital Militar. Igualmente, fue solidaria durante el conflicto con Ecuador, en el año 1910, pues organizó la «Unión Patriótica de Señoras». En el Perú, había alta mortalidad materno-infantil y por tuberculosis. Investigó sobre la tuberculosis y presentó una ponencia al respecto en el $\mathrm{V}$ Congreso Médico Latinoamericano celebrado en Lima, en 1913: "La necesidad de la declaración obligatoria de la tuberculosis pulmonar y del establecimiento de sanatorios por el Estado». Contribuyó así al desarrollo de la salud pública nacional ${ }^{3}$ ).

La Doctora Laura Esther Rodríguez fue médica de la Escuela Normal de Mujeres, del Liceo Fanning, de los Conventos de La Concepción, Jesús María y Nazarenas.

Infortunadamente, cuando todavía se esperaba mayores contribuciones suyas a la vida nacional, la pionera médica peruana, después de padecer una prolongada enfermedad, falleció en Lima el 6 de julio de 1919, a la edad de 46 años.

Sin lugar a dudas, el fulgor de su ejemplo y la dignidad que supo dar a su quehacer profesional, constituyen un paradigma para la mujer peruana. Ella fue médica, clínica, docente, investigadora y líder de la comunidad. Delineó las rutas, tal vez sin advertirlo plenamente, en las cuales las venideras médicas peruanas habrían de continuarla en el ejercicio de la profesión médica. Ella dio el primer paso para lograr la común unión participativa de la mujer en la vida nacional, en igualdad de condiciones. También, contribuyó eficazmente al bienestar y desarrollo de la salud pública nacional.
La Beneficencia Pública le erigió un busto en el Parque de la Historia de la Medicina Peruana, con una placa recordatoria, en Lima, en el año 1972, donde decía: «Placa Recordatoria a la Dra. Laura Esther Rodríguez Dulanto. 1876-1919. UNMSM 22-IX-1900». Hoy, su nombre perdura en el Hospital MINSA de su pueblo natal Supe $\left({ }^{8}\right)$. En Lima es homenajeada por la Asociación Cultural Vidal Hijos de Supe y por su Alma Mater.

\section{AGRADECIMIENTO}

Al señor Carlos Giovanni La Rosa Benedicto; por su valiosa colaboración para hallar la partida bautismal de mi paisana, la Dra. María Laura Esther Rodríguez Dulanto.

\section{REFERENCIAS BIBLIOGRÁFICAS}

1. La Primera médica peruana. Diario El Comercio. 12 de marzo de 1995.

2. De los Ríos, E. Una bella historia: Laura Rodríguez Dulanto. Revista Caretas. 5 de febrero de 1990. p. 54-5.

3. Alayza F. La mujer y la medicina peruana. Acta Médica Peruana. 1991;15(2):70-3.

4. Delgado G, Chara R. Dra. Laura Esther Rodríguez Dulanto (Supe 1875 - Lima 1919). En: Delgado G, Chara R (eds). Evolución Histórica de la Facultad de Medicina de San Fernando de la UNMSM. Sesquicentenario de la Facultad de Medicina, 1856-2006. Lima: UNMSM; 2006:121-2.

5. Delgado G. Laura Esther Rodríguez Dulanto (1876-1919). En: Historia de la Medicina Peruana en el Siglo XIX. Tomo II. Lima: UNMSM; 2000:1278-9.

6. Triunfa la mujer peruana. Bachiller en Ciencias (27-X-1898). Será médica. Diario El Comercio. 28 de octubre de 1998.

7. Lastres J. Historia de la Medicina Peruana. Tomo III. Lima: UNMSM; 1951. p. 300.

8. Resolución Directoral N²96-DG-DESP-DISA-III-LN-2005 del 21 de junio del 2005 se otorga la Categoría II-1 al Hospital de Supe "Laura Esther Rodríguez Dulanto" del ámbito jurisdiccional de la Dirección de la Red de Salud. Lima Norte 1 - Dirección de Salud III Lima. Diario El Peruano. 13de febrero de 2006.

Manuscrito recibido el 20 de julio de 2007 y aceptado para publicación el 28 de agosto de 2007.

Correspondencia:

Héctor Díaz Núñez

Servicio de Gastroenterología

Hospital Nacional Guillermo Almenara Irigoyen

Av. Grau 800. Lima 1, Perú

Correo-e: anales@unmsm.edu.pe 